under a flow of $10 \% \mathrm{H}_{2} / \mathrm{F}_{2}$ to produce black powders of Pd@MCM-48 and Pd@SBA-15. X-ray diffraction and transmission electron microscopy (TEM) analysis show that the Pd is distributed locally inside the pores and does not block the channel entrances. The sizes of the ballshaped Pd domains obtained have an average diameter of $35-40 \mathrm{~nm}$. They are connected in a three-dimensional network, that is, the Pd domains fill the pores in the cubic matrix of MCM-48 with Ia3d symmetry. To verify that the Pd domains were free and the major product of the experiment, after the Pd@MCM-48 was treated with $20 \% \mathrm{HF}$ to dissolve the silicate matrix, TEM analysis showed that "the Pd ball-shaped domains consist of three-dimensionally interconnected networks" whose shape and pores are "a replication of the MCM-48 template," according to the article. Using the same method, Pd nanowires were obtained. SBA-15 was used as a template, resulting in 150-nm ellipsoidal domains with average diameters of $9 \mathrm{~nm}$. This is almost the same as the diameter of the channel in the SBA-15 matrix.

IULIA MunTELE

\section{Low-Strain Warm Pressing of Multilayer Composites Yields Layers with Longer-Range Protection from Crack Deflection}

To obtain a strong material with good crack-deflection properties, a delicate balancing act between a strong matrix material and a weak, crack-blunting second phase is required. In a study conducted by E.J. Winn and I.-W. Chen of the Department of Materials Science and Engineering at the University of Pennsylvania, crackpropagation mechanisms of zirconiabased composites containing an alumina second phase were examined. Despite the presence of a second phase, the samples demonstrated high bending strengths.

As reported in the December 2000 issue of the Journal of the American Ceramics Society, the two sample types used in the experiments had identical configurations. They were composed of 16 zirconia matrix layers, with 15 alumina (glue) interlayers. The matrix element was fabricated with zirconia slurries cast into strips of tape $100 \mu \mathrm{m}$ thick. The interlayer glue consists of a porous alumina solution. A type of polymer known as AST, or alkalisoluble swellable thickener, was added to a mixture of alumina powder and acidic water $(\mathrm{pH} 4)$. While stirring, the $\mathrm{pH}$ of the mixture slowly changes to 9 with $\mathrm{NaOH}$. The change in $\mathrm{pH}$ causes the AST to expand, adding volume but not weight. Subsequent sintering causes the AST to disintegrate, leaving large pores behind. The layered samples were assembled by stacking 1.5 in. $\times 1$ in. $\times 100 \mu \mathrm{m}$ layers of zirconia tapes with a thin layer of glue between them.

The first sample was fabricated from the above formula and warm-pressed at $90^{\circ} \mathrm{C}$ to $20 \%$ of its true strain value. The second sample was prepared by pressing at the same temperature, but to $100 \%$ of its true strain value. Both samples were sintered for $30 \mathrm{~min}$ at $1350^{\circ} \mathrm{C}$. Polished samples were subjected to the three-point bend test at $0.2 \mathrm{~mm} / \mathrm{min}$ displacement. The mean strengths recorded for the two samples are $580 \mathrm{MPa}$ and $540 \mathrm{MPa}$, respectively.

Examination of cross-sectional micrographs of the two samples suggests that through thickness, cracks were more likely to result from sample type 2 . High strain from the press caused the interlayers to buckle and break apart. Thus, it is possible for cracks to "find paths through the material which never intersect a second-phase region," as reported by the researchers. In sample type 1 , the interlayers remained mostly straight and unaltered. This offered longer-range crack-deflection protection than found in the type 2 samples. In both cases, however, the chief mechanism of crack propagation was along the alumina/zirconia interface. These cracks ran perpendicular to the direction of applied stress, cleaving the interfaces.

These experiments supported the formation of composites with variable crack-deflection proficiencies while only marginally compromising the strength of the bulk material.

JUNE LAU

\section{Simulation Tool Demonstrates Double-Gate Transistor with 10-nm-Long Electrodes}

Using a simulation tool called nanoMOS, engineers at Purdue University have shown that a double-gate transistor carries twice the electrical current and could work more than twice as fast as conventional devices. The research team led by Mark Lundstrom and Supriyo Datta, professors of electrical and computer engineering at Purdue, said that this type of transistor could keep Moore's law in force until 2025 or beyond. This would give scientists time to develop technologies to replace integrated circuits made from silicon.

As described during the International Electron Devices Meeting, sponsored by the Institute of Electrical and Electronics Engineers in San Francisco on December 13, the electrodes known as gates are $10 \mathrm{~nm}$ long, rather than $100 \mathrm{~nm}$ as in conventional transistors.

The researchers have made nanoMOS available through the Purdue Nanotechnology Simulation Hub, or nanoHub. Lundstrom said that the nanoHub uses a network-computing platform that automatically enables computer users to run programs with conventional Web browsers. The nanoHub can be accessed at www.nanohub.purdue.edu.

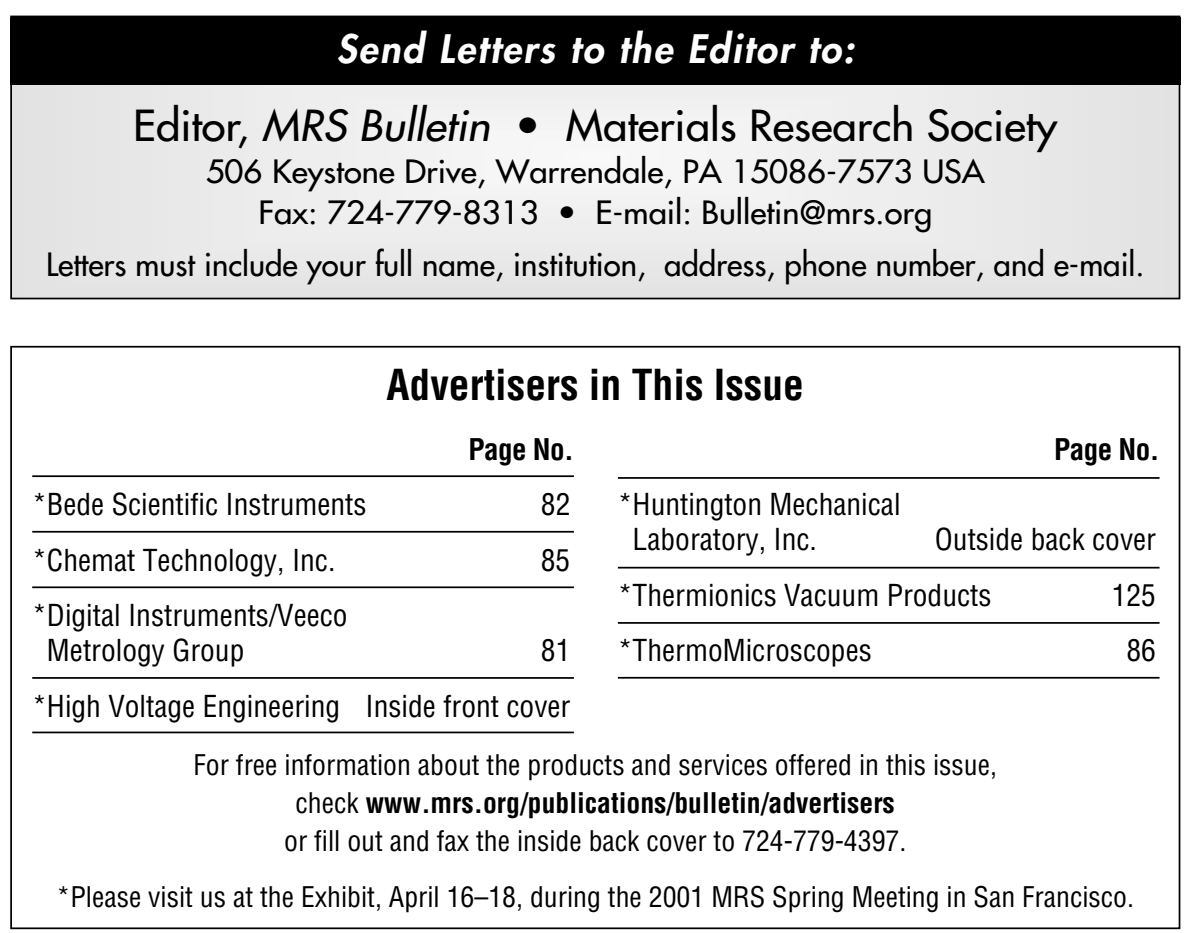

